\title{
Diffraction Line Width in Quasicrystals-Sharper than Crystals
}

\author{
Antony J. Bourdillon \\ UHRL, San Jose, CA, USA \\ Email: bourdillona@xraylithography.us,www.xraylithography.us
}

Received 7 July 2016; accepted 28 August 2016; published 31 August 2016

Copyright (C) 2016 by author and Scientific Research Publishing Inc.

This work is licensed under the Creative Commons Attribution International License (CC BY). http://creativecommons.org/licenses/by/4.0/

(c) (i) Open Access

\section{Abstract}

A quasicrystal has a structure intermediate between crystals and compound glasses. The disorder in glass makes its diffraction diffuse, so it is surprising that quasicrystals diffract more sharply than crystals. The greater sharpness is computed to be due to the hierarchic structure with unit cell alignment in 3-dimensional space. Electron microscope phase contrast images map the comparatively heavy $\mathrm{Mn}$ atoms in icosahedral $\mathrm{Al}_{6} \mathrm{Mn}$, where the transition metal locates the centers of unit cells inside clusters and superclusters. Because the solid is aperiodic, each diffracted beam is a product of multiple interplanar spacings combined, and this contrasts with the unique relationship between spacing and incident angle in Bragg diffraction from crystals. Simulated quasistructure factors add the relative phase shifts that are in geometric series from cell to cluster to superclusters of increasing order. The scattering becomes coherent in best fit, angular configuration between the aperiodic solid and a longitudinally periodic X-ray or electron probe. The quasistructure factors express angular divergence in each diffracted beam from its corresponding Bragg condition, and the divergence provides a special metric, essential for atomic measurement in the geometric solids. The fit is reinforced at all levels from the unit cell to cluster to high order superclusters. The optics operates under a new quasi-Bragg law in a new geometric space. In this paper, we proceed to examine the effect of specimen size on line resolution in diffraction, first analytically and secondly in simulation. The line resolution follows a power law on the supercluster order, matching its atomic population.

\section{Keywords}

Quasicrystal, Line Width, Quasi-Structure Factor, Geometric Space, Hierarchic, Metric

\section{Introduction}

Prima facie, an aperiodic solid should scatter incoherently or diffusely, as from an amorphous material or a gas. 
In these, bond length information can be obtained by measuring Bragg angles observed in coarse patterns with cylindrical symmetry about the incident probe. The quasicrystalline, icosahedral phase, $i-A l_{6} M n$, was discovered thirty four years ago. It has a sharp diffraction pattern containing five-fold axial symmetries and an aperiodic atomic map. The quasicrystal does not belong to any member of the complete set of fourteen Bravais lattices that contain all crystals. These comprise unit cells that fill space with face sharing surfaces. Nine years ago, Senechal wrote for the American Mathematical Society the paper, "What is a quasicrystal?" It began, "The short answer is no one is sure [1]". Since then, the structure has become clear by consistent interpretation of phase contrast micrographs [2]-[7] together with understanding of the 3-dimensional diffraction patterns [4] [8], and consistent measurement at the atomic scale.

Like fused silica, quasicrystals have unit cells, as imaging shows, and these are likewise edge sharing, but they differ from the glasses because the unit cells in the quasicrystal are uniformly oriented due to multiple edge sharing. The structure is hierarchic so that the diffraction and many other physical properties can be calculated easily. The diffraction does not follow Bragg's law of diffraction for crystals for many reasons, the most obvious being that the diffraction series are not in linear order; they are in 3-dimensional geometric series [3] [4]. Moreover, because the solids are aperiodic, the diffraction of a single beam from a quasicrystal is due not to a single, specular, diffraction plane selected by precise crystal orientation, but is due to many planes of atoms, at various planar separations ( $d$ spacings) scattering simultaneously. Each scattered beam results from an effective interplanar spacing which is a compromise between many real interplanar spacings. Likewise the diffraction angle is a compromise that is not given by Bragg's law. In quasicrystals, the diffraction follows a quasi-Bragg law that describes the geometric series that is observed [6] [9]. With both of these compromises in the aperiodic material, a special metric is needed to derive atomic measurements from the diffraction. This metric is calculated through simulated quasi structure factors. We have shown previously how quasi-structure factors are calculated for large quasicrystals [6] [10]; here we show why diffracted beams have to be sharper in ideal quasicrystals than diffraction from perfect crystals.

\section{Structure}

Figure 1 shows, at left, a micrograph of $\mathrm{Al}_{6} \mathrm{Mn}$ obtained at optimum defocus in phase contrast electron microscopy [2] [3]. Each circular spot maps a $M n$ atom. It is located at the centre of a unit cell and the atom is surrounded by 12 extremely tightly bound $\mathrm{Al}$ atoms. The tight binding depends on the precise ratio of atomic diameters in the metal alloy:

$$
\frac{d_{\text {solute }}}{d_{\text {solvent }}}=\sqrt{1+\tau^{2}}-1
$$

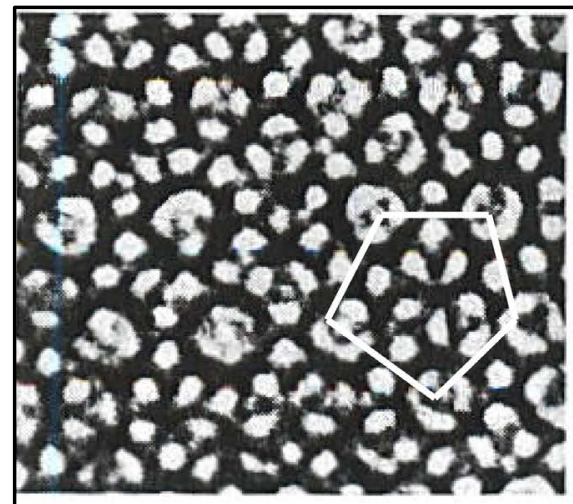

(a)

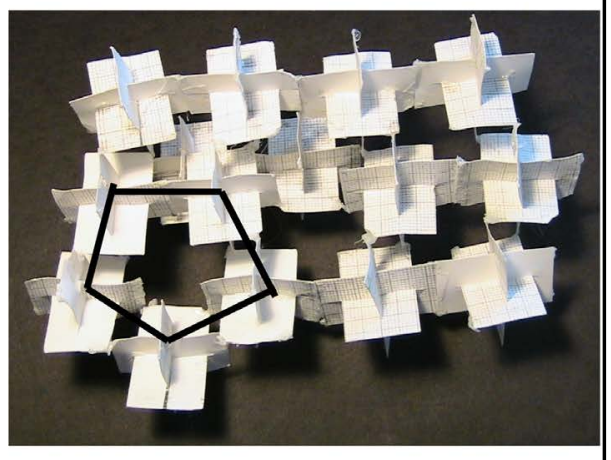

(b)

Figure 1. (a) Optimum defocus electron microscope image of $A l_{6} M n$ [2] [3] (reprinted with permissions from L. A. Bursill, J. L. Peng and from Nature). (b) In mirror image, skeletal structures, representing icosahedral clusters, match the micrograph. As a cluster, each corner of the golden rectangular triads locates an icosahedral unit cell of one central $\mathrm{Mn}$ surrounded by $12 \mathrm{Al}$ atoms. Note the sections of supercluster order 1 outlined in each image with pentagons that connect cluster centres. The unit cells are edge sharing and aligned. 
where the golden section, $\tau=(1+\sqrt{5}) / 2$. The ratio is found generally in diatomic quasicrystals. The micrograph maps cluster sections as circles of 10 unit cells. The white pentagon connects five clusters in a section of a supercluster. The clusters are represented, at right, by golden triads formed from golden rectangles, each with length to width ratio $\tau$. The golden triad is a skeleton for an icosahedron, having 12 corners that connect 20 triangular faces with 30 congruent edges. On scales that vary by the stretching factor $\tau^{2}$, the triads may also be used to represent unit cells or any order of supercluster in the hierarchic structure.

\section{The Metric $c_{s}$ that Relates Structure to Diffraction Angle}

Since the quasicrystal diffraction pattern does not follow Bragg's law, how are measurements to be made at the atomic scale? The logarithmically periodic solid (LPS) [6] has many advantages: measurement is its greatest. Calculations of structure factors for the LPS demonstrate that they are all approximately zero: there is no Bragg diffraction, and this is a consequence of aperiodicity. In each single quasi-Bragg reflection, multiple interplanar spacings operate - contrasting with crystals where the spacing is unique for each reflection. However by scanning over the scattering angle, a compromise scattering angle is found at best fit. A quasi-Bloch wave for this fit is illustrated in Figure $2[6]$. The quasi-Bloch wave peaks do not coincide with atomic planes ${ }^{1}$.

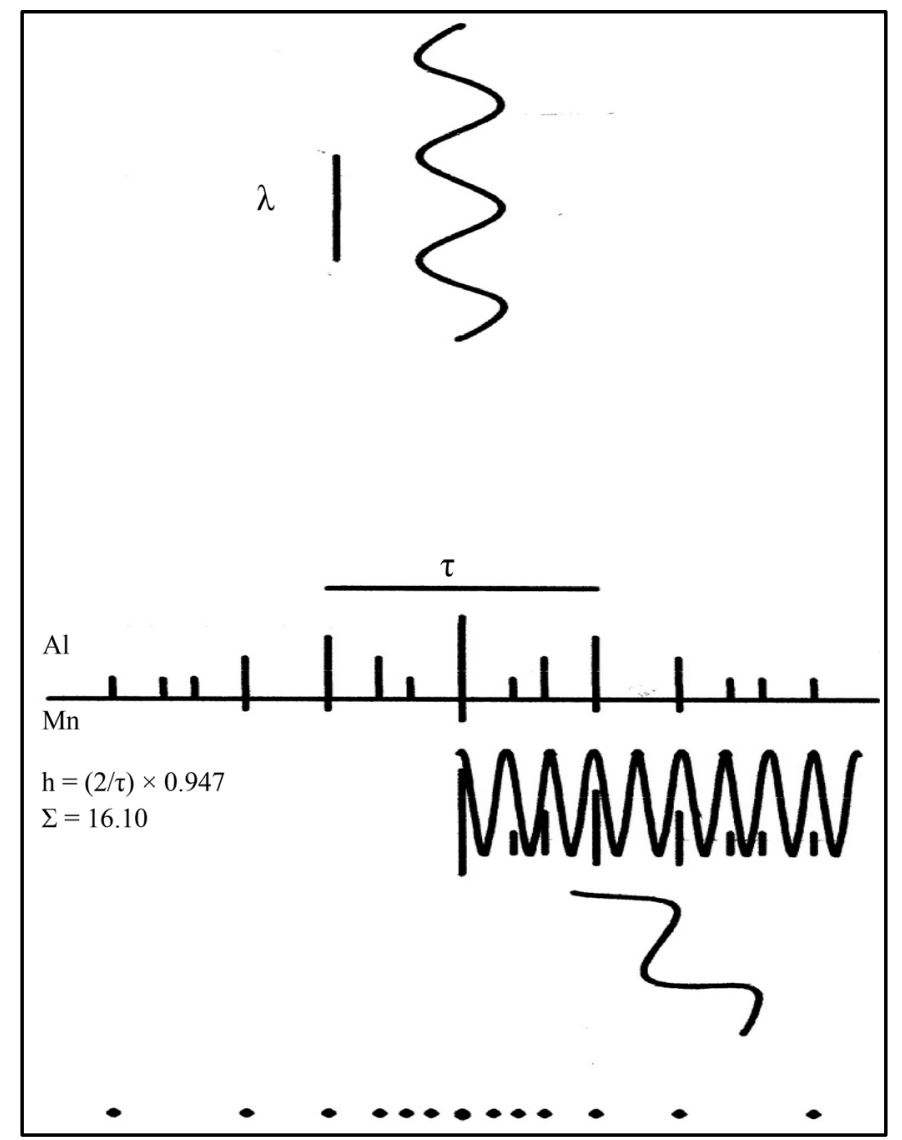

Figure 2. A periodic electron beam, moving downwards, scatters from atomic planes on an aperiodic quasicrystal cluster to form a diffraction pattern in geometric space. This is due to a quasi-Bloch wave having maximum overlap with the populations on the atomic planes ([6] reprinted with permission). Al atomic populations lie above the abscissa; $M n$ atoms below, as shown. The maximum overlap occurs at the quasi-Bragg angle $c_{s} \theta_{B}$, where $\theta_{B}$ would be the crystalline Bragg angle for diffraction from a corresponding unique interplanar spacing such as $1 / \tau$, or $1, \tau, \tau^{2}$ etc.

\footnotetext{
${ }^{1}$ High resolution transmission electron microscopy does not image atoms; the Fourier transform method of analysis is misleading.
} 
Moreover, this scattering angle maximizes only in second Bragg order, $n=2$; The linear Bragg orders, 1, 3, 4, $5 \cdots$ are forbidden owing to approximately half integral values in the geometric series $1 / \tau$, or $1, \tau, \tau^{2}[3][4]^{2}$. Without this restriction the diffraction pattern would not be geometric, as observed. Dividing iteratively by the scaling stretching factor $\tau^{2}$, the half integrals repeat throughout the geometric series. The maximum in the quasi-scattering factor is found at a quasi Bragg angle $\theta^{\prime}=c_{s} \theta_{B}$, where $c_{s}$ is what we called the compromise spacing effect and $\theta_{B}=\sin ^{-1}(n \lambda / 2 d)$ the corresponding angle under Bragg's law for an interplanar spacing $d$. The wavelength of the X-ray or electron beam is $\lambda$. Computations show that $c_{s}$ has the same value for all quasi-Bragg diffracted beams, and that value is about 0.947 , so that $\theta^{\prime}$ is $5.3 \%$ less than $\theta_{B}$. The simulated compromise spacing effect has a value close to the intuitive value $2.5 / \tau^{2}$, and is employed in the following section. The numerator is the nearest half integral to the denominator, which is the stretching factor between hierarchic orders. With these adjustments the quasi-Bragg law may be written:

$$
\tau^{m} \lambda=d^{\prime} \sin \left(c_{s} \theta\right)
$$

with order $-\infty<m<\infty$. Computations show that $c_{s}$ is the same for all $m$ and all indexed reflections. Notice that every term in the quasi-Bragg law is different from every term in Bragg's law excepting only the wavelength. While aperiodicity requires a new law in physics, the geometric diffraction defines a new space.

\section{Computations on Quasi-Scattering Factors}

\subsection{The Metric $c_{s}$, That Relates Structure to Diffraction angle}

Quasi-scattering factors $F_{h k l}$ were calculated using the corresponding standard formula in crystallography [11] for a cebtrosymmetric crystal, adapted with the factor $c_{s}$ :

$$
F_{h k l}=\sum_{i=1}^{N} f_{i} \cos \left(2 \pi \cdot c_{s}\left(\overline{h_{h k l}} \cdot \overline{r_{i}}\right)\right)
$$

summed over all $N$ atoms in a truncated hierarchic quasicrystal. The factor modifies the projections of the atoms onto a scattering plane normal $\overline{h_{h k l}}$ having indices $h, k, l$. The atomic scattering factors $f_{i}$ are appropriate for either $A l$ or $M n$ and are found in tables [12] [13]. The LPS is centro-symmetric.

In large quasicrystals, computation of Equation 3 is restricted by truncation errors. However, the number of computations is reduced, without sacrifice of accuracy, by calculating quasi-structure factors iteratively from unit cell to cluster to superclusters of increasing order $p[10]$ :

$$
F_{h k l}^{p}=\sum_{c c=1}^{12} \cos \left(2 \pi \cdot c_{s}\left(\overline{h_{h k l}} \cdot \tau^{2 p} \overline{r_{c c}}\right)\right) F_{h k l}^{p-1}
$$

where $\overline{r_{c c}}$ describes the 12 vectors to cluster centers [10], and $F_{h k l}^{\text {cluster }}$ may be written $F_{h k l}^{0}$ :

$$
F_{h k l}^{\text {cluster }}=\sum_{c c=1}^{12} \cos \left(2 \pi \cdot c_{s}\left(\overline{h_{h k l}} \cdot \overline{r_{c c}}\right)\right) F_{h k l}^{\text {cell }}
$$

while $F_{h k l}^{\text {cell }}$ uses Equation (3) to sum over the 13 atoms in the unit cell. In these calculations care is taken to halve the scattering factors in atoms, in clusters or supercluster of whatever order, that are counted twice; or to divide by three times on atoms counted thrice (Appendix). These Equations (1) to (5) have been previously described in greater detail than is given here in summary. The chief purpose of this paper is to demonstrate how the sharpness of the diffraction pattern is represented by these structure factors. Now we proceed to a mathematical representation of resolution by an application of second derivatives in hierarchic arrangement. To prove it, we shall compute the dependence of $c_{s}$ on specimen size, and then its dependence on both short range (unit cell) or long range (lattice) symmetries ${ }^{3}$. A differential formula is used to express resolution, or line width. The formula itself matches the computations made on larger quasicrystals, and provides confidence that the sharp diffraction is completely understood in these wonderful, but no longer so new materials. Clearly, since the diffraction does not follow Bragg's law, there are many aspects that we cannot take for granted; one is the sharpness of the diffraction. The theoretical understanding will make it possible to interpret, in various samples, the effect of defects, espe-

\footnotetext{
${ }^{2}$ In icosahedral units the unit cell has length, $a=\tau$; and edge width unity. The conversion to SI units is given in [4].

${ }^{3}$ The terms in the geometric series tend to integral values at high order [3]. The overlap with the linear, Bragg series has no effect on $c_{s}$.
} 
cially in rapidly quenched material. One of the defects is truncation of the ideal structure that can, in principle, be estimated from the results presented here. The simulations described in this paper take no account of thermal effects, normally represented in crystallography by the Debye-Waller factor, nor do the simulations account for absorption and lesser effects.

\subsection{Hierarchic Computation of Resolution}

We begin with two hypotheses. The first is that the profile that is given by scanning the value of $c_{s}$ in Equation (4) is approximately Gaussian as in the normal distribution. This hypothesis is suggested by the requirements for the best fit illustrated in Figure 2 and is indicated in the following simulations. The second hypothesis is that there exists a resolution function $f\left(c_{s}\right)$, that is approximately the same for all levels of unit cell, cluster and orders of supercluster. This hypothesis is supported by the repeating features of the geometric series (ratio $\tau$ ) scaled between orders by the stretching factor $\tau^{2}$. To fix ideas, we suppose that the resolution function depends on the second derivative of the profile, at its peak (where the first derivative is zero), of the scan in $c_{s}$. A Gaussian profile, $f=A \exp \left(-\left(c_{s}-c_{p}\right)^{2} / 2 s^{2}\right)$, will then yield the second derivative as follows:

$$
\begin{gathered}
\frac{\partial f}{\partial c_{s}}=-A \frac{2\left(c_{s}-c_{p}\right)}{2 \sigma^{2}} \exp \left(-\frac{\left(c_{s}-c_{p}\right)^{2}}{2 \sigma^{2}}\right) \\
\left.f^{\prime \prime}\right|_{c_{s}=c_{p}}=-\frac{A}{\sigma^{2}}
\end{gathered}
$$

The metric is the mean value of $c_{s}$ in the peak profile: the value that is scanned in Equation (3), gives the metric that maximizes at $c_{p}$, since the profile is symmetric. The least squares linear fit, as illustrated in Figure 2, does not provide an analytical solution for $A$ and $\sigma$, but they can be assessed in the computational scans recorded below. Suppose the resolution is some product $B \cdot f^{\prime \prime}\left(c_{s}\right)$. The iterative nature of Equation (5) will then give for the resolution $R$ of a supercluster order $p$, a value $\left(B \cdot f^{\prime \prime}\left(c_{s}\right)\right)^{p}$.

$$
R_{h k l}^{p}=\left(B \cdot f^{\prime \prime}\left(c_{s}\right)\right)^{p}
$$

Since $p=\log \left(R_{p h k l} /\left(B f^{\prime \prime}\left(c_{s}\right)\right)\right)$, on a logarithmic scale this resolution dependence on $p$ should plot onto a straight line.

This dependence would be consistent with a dependence on the number of scattering atoms which has approximately the same power law dependence on the order of supercluster. Where the unit cell has 13 atoms, some of them shared with neighboring atoms, the cluster contains 12 unit cells, again with some atoms shared. The number of scattering atoms increases by about an order of magnitude with each increase in order, the cluster being supercluster order 0, with about 100 atoms. Further details are given in Appendix.

\subsection{Dependence of $c_{s}$ on Specimen Size and Range}

The diffraction in quasicrystals is not Bragg diffraction and the structure factors demonstrate the fact. Quasistructure factors provide the value for $c_{s}$, the metric which is necessary for atomic scale measurements in the solids. The following two figures have previously been discussed in detail [14] so we give here the conclusions in summary form. Figure 3 is a $\log$ plot of quasi-structure factors calculated by scanning $c_{s}$ in Equation (5) [6]. The reflection peak shown is typical and is here calculated for the $(2 / \tau, 0,0)$ reflection [3] [4] from a supercluster order 6. The fractional divergence from the Bragg position $\left(\theta_{B}=\sin ^{-1}(n \lambda / 2 d)\right)$ is $c_{s}=0.947($ or $1.0-534 \times$ $0.0001)$. The quasi-Bragg angle $\theta^{\prime}$ is $5.3 \%$ smaller than the equivalent Bragg angle $\theta_{B}$ for crystals on a corresponding interplanar spacing, such as $d=\tau^{-1}, 1, \tau, \tau^{2}$ etc. In consequence, the measured quasi-spacing $d^{\prime}$ is $5.3 \%$ larger than would be given by using Bragg's formula, $d=n \lambda / 2 \sin (\theta)$. The difference is the compromise effect due to logarithmic periodicity and gives the value for the metric $c_{s}$ at the peak of the computational scan. The simulated metric is found to have the same value in all quasi-Bragg reflected beams. It provides consistent values for atomic size, cell size, cluster size etc. The Bragg angle corresponds to channel 1 in the plot. All struc- 


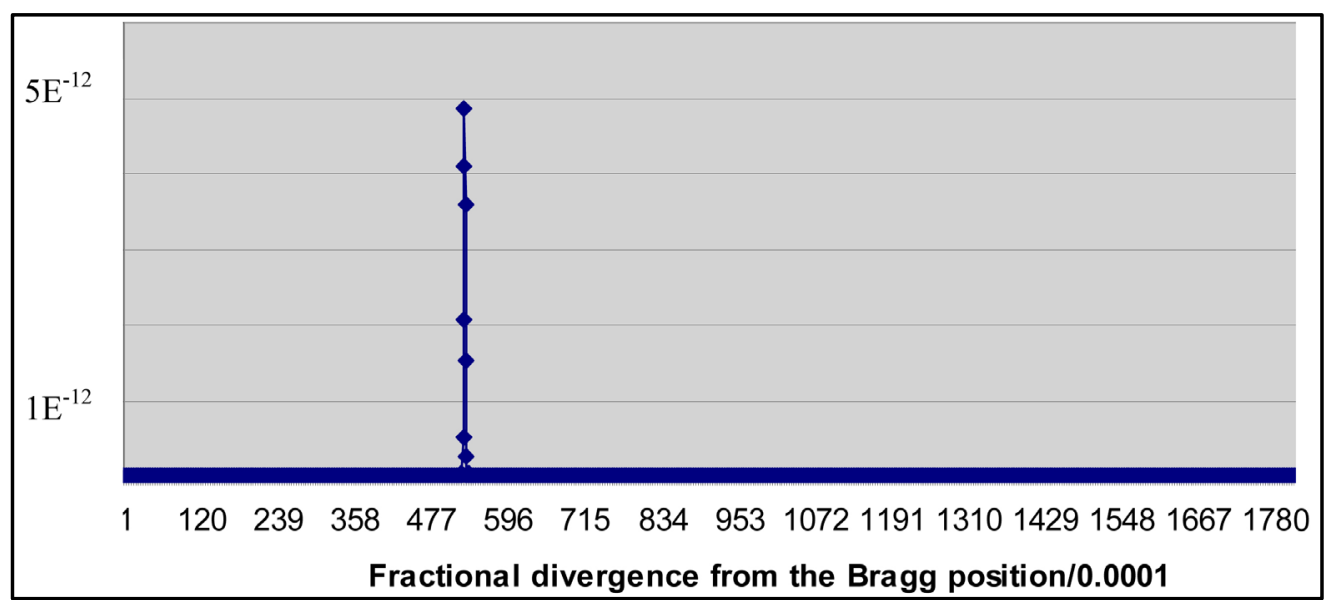

Figure 3. Simulated quasi-structure factors, scanned away from the Bragg angle for the reflection $(2 / \tau, 0,0)<c_{s}<0.8(2 / \tau, 0,0)$. The calculation is for a supercluster order 6 . Fractional divergence is zero at the nominal Bragg angle on channel 1 [6] (reprinted with permission from Nova Science Publishers).

ture factors (as for Bragg's law) are zero. The ordinate values compare with the square of the number of atoms in the supercluster, the atomic scattering factors $f_{i}$ being of order 1 (see Appendix).

Does $c_{s}$ change as the cluster size is increased or decreased? The computations shown in Figure 4(a) were performed on superclusters orders 0, 1, 2 and 3, using the method of Equations (3) - (5). The value of the metric $c_{s}$ at the peak of the scan is constant; while there is a general decrease in line width as the order increases. The figure shows simulated profiles for diffracted beams from the hierarchic icosahedral structure. This is compared with three computational devices: in Figure 4(b) the structure factor profile is calculated for the face centered cubic (fcc) structure. Notice that the peak occurs at the Bragg angle (channel 1). Its width is more than double the width of the profile for the supercluster order 2 (Figure 4(a)), having an equivalent number of atoms. This fact implies stronger coherence in the logarithmic solid. Figure 4(c) and Figure 4(d) are computational devices on unrealistic structures: in the former case an fcc unit cell is placed in an icosahedral lattice; in the latter an icosahedral cell is placed in a cubic lattice. These cases have similar profiles with similar $c_{s}$ offsets. They demonstrate that the offsets are due to the geometric series whether it occurs in the cell or in the lattice, and that the influence of the long range lattice is the same as the influence of the short range unit cell. Both filter-in the offset and filter-out Bragg diffraction. This computational fact is consistent with the repeated series of spacings, scaled between supercluster orders by the ratio $\tau$ in the geometric series and by the stretching factor $\tau^{2}$ in the hierarchic structure.

In Figure 5, the logarithms of simulated line widths in Figure 3 and Figure 4(a) are plotted against supercluster orders. The straight line fit is predicted by the power law in Equation (7). This least squares fit and these graphs confirm consistently the origin of both the compromise spacing effect and the line widths, i.e. the hierarchic structure and geometric series diffraction. The least squares, linear fit to the computation is $\log$ (half width $)=-0.96(3)-0.42(7) p$ as illustrated in the figure.

Experimental measurement of line width is complicated by the restricted dynamic range of CCD (charge coupled device) detectors and of camera plate. Moreover, most diatomic quasicrystals are dual phase with fine structures, or metastable so that large crystals are grown with difficulty. We therefore leave for another time the various ways in which line width might be measured in specimens prepared under particular conditions. However measured, divergence of resolution, or line width, from the linear fit shown in the figure could be used to measure defects or defect concentrations, including truncations of the hierarchic structure.

\section{Discussion}

What new properties do these comparatively new materials have? While they share some properties in common with metallic glasses, such as corrosion resistance [15] they have special electronic, magnetic and mechanical properties associated with their geometric electronic band structures [5] [14]. Their most fundamental property is the one that is analyzed here because most of the other properties follow from it: how it comes about that an aperiodic material can diffract with a sharp pattern in geometric series? Besides the new quasi-Bragg law and new 


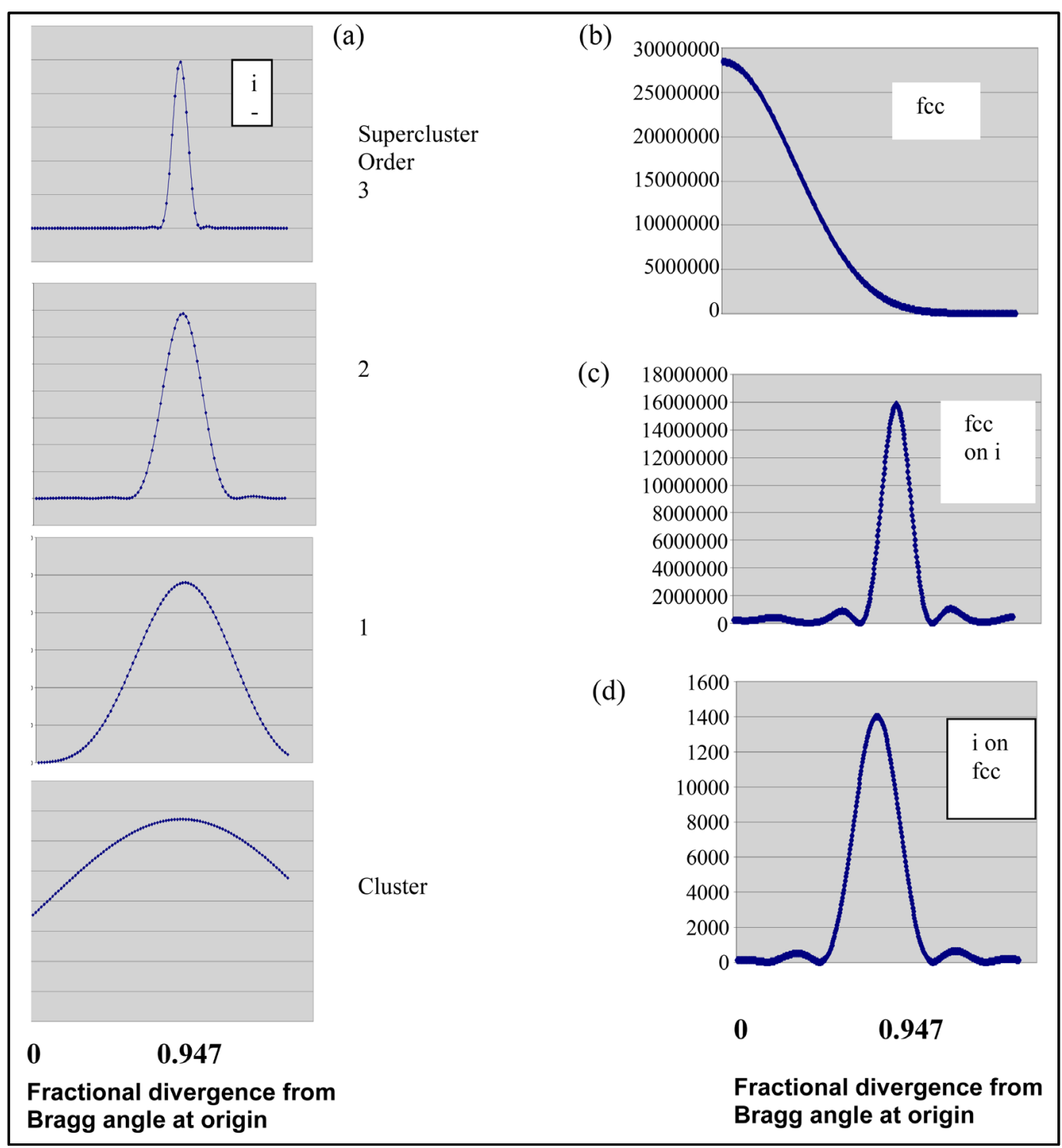

Figure 4. (a). Computed intensities, i.e. squared structure factors, for $(2 / \tau, 0,0)$ diffraction in supercluster orders $3,2,1$, and in an $\mathrm{i}-\mathrm{Al}_{6} \mathrm{Mn}$ cluster. The intensities are plotted against divergence from Bragg angle and span $\sim 15 \%$ of this angle. The Compromise Spacing Effect (CSE) shifts quasi-Bragg angle from the calculated Bragg angle by $5.3 \%$. The lines from different supercluster orders show the same $c_{s}$ displacement from the Bragg angle. (b) (111) structure factors, computed against angle as above, for a cubic cluster of fcc Al, having about 19,000 atom sites, similar to quasicrystal supercluster order 2. There is no CSE. At this size, the peaks are computed to be comparatively broad, typically $5 \%$ of the Bragg angle. (c) Computed structure factor for (111) diffraction by an imaginary cubic fcc cell in a logarithmically periodic icosahedral grid, as in supercluster order 2. The CSE occurs with a peak narrower than in Figure 4(b) for the crystalline structure. (d) Computed structure factor for $(\tau \tau \tau)$ i-Al ${ }_{6} \mathrm{Mn}$ icosahedral cell in an imaginary cubic grid of side $\tau$ and site population about 20,000 (like (b) and (c)).

geometric space, the hierarchy provides, theoretically, higher sharpness and coherence than does the periodicity of crystals.

The fact also suggests a possible extension of finite element analysis. On reflection, it is very surprising that the hierarchic structure produces greater coherence in geometric space than do crystals in linear space. It might prove possible to eliminate subsidiary minima or maxima by overlaying geometric analysis on conventional linear calculations. The stretching factor, that is strict in the present model, might be replaced by diffusion 


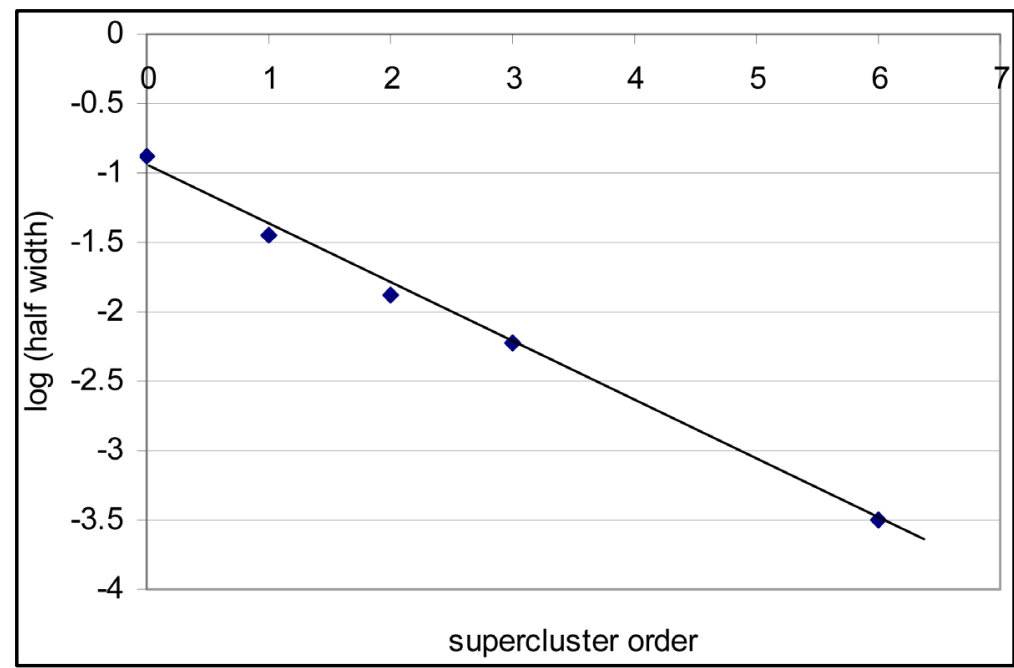

Figure 5. Logarithm of half widths shown in Figure 3 and Figure 4(a) plotted against supercluster order, compared with least squares linear fit. The straight line was predicted in Equation (7).

coefficient, thermal conductivity, viscosity coefficient, or other physical parameter. These extensive influences expressed logarithmically in either initial conditions or by progressive computation, might be found to accelerate convergence and to eliminate subsidiary solutions.

\section{Conclusion}

A mathematical treatment of the geometric relationships inherent in the ideal hierarchic structure has been applied to examine sharpness in quasicrystal diffraction. It is not only sharp, but in principle, sharper than in perfect crystals. These calculations ignore the blurring effects of defects, which may be expected to depend on materials processing routes. However, they also suggest methods for characterizing the defects. More generally, the results, showing greater sharpness in geometric space than in linearly periodic crystals, suggest opportunities for both optics and for general computational methods in finite element analysis.

\section{References}

[1] Senechal, M. (2006) Notices to the American Mathematical Society, 3, 886-887.

[2] Bursill, L.A. and Peng, J.L. (1985) Nature, 316, 50-51. http://dx.doi.org/10.1038/316050a0

[3] Bourdillon, A.J. (2012) Metric, Myth and Quasicrystals. UHRL, ISBN 978-0-9789-8393-2. https://www.youtube.com/watch?v=xD30KF93qio

[4] Bourdillon, A.J. (2009) Quasicrystals and Quasi Drivers. UHRL, USA, ISBN 978-1-4389-5589-6.

[5] Bourdillon, A.J. (2009) Solid State Communications, 149, 1221-1225. http://dx.doi.org/10.1016/j.ssc.2009.04.032

[6] Bourdillon, A.J. (2011) Logarithmically Periodic Solids. Nova Science, USA, ISBN 978-1-61122-977-6. Bourdillon, A.J. (2010) Youtube/Quasicrystals, Logarithmically Periodic.

https://www.youtube.com/watch?v=A6vpsWv9nsQ

[7] Bourdillon, A.J. (2014) Journal of Modern Physics, 5, 488-496. http://dx.doi.org/10.4236/jmp.2014.56060

[8] Bourdillon, A.J. (2013) Micron, 51, 21-25. http://dx.doi.org/10.1016/j.micron.2013.06.004

[9] Bourdillon, A.J. (2014) Journal of Modern Physics, 5, 1079-1084. Bourdillon, A.J. (2015) Youtube/Log-Lin Metric in Quasicrystals. http://dx.doi.org/10.4236/jmp.2014.512109

[10] Bourdillon, A.J. (2016) Journal of Modern Physics, 7, 43-50. http://dx.doi.org/10.4236/jmp.2016.71005

[11] Cullity, B.D. (1978) Elements of X-Ray Diffraction. Addison-Wesley, Boston.

[12] Hirsch, P., Howie, A., Nicholson, R.B., Pashley, D.W. and Whelan, M.J. (1977) Electron Microscopy of Thin Crystals. Krieger. 
[13] Doyle, P.A. and Turner, P.S. (1968) Acta Crystallogr, 24, 39.

[14] Bourdillon, A.J. (2010) Quasicrystals' 2D Tiles in 3D Superclusters. Author House, ISBN 978-1-4490-8395-3.

[15] Puckerman, B.E. (2011) Quasicrystals, Types, Systems and Techniques. Nova, New York.

[16] Pauling, L. (1985) Letters to Nature, 317, 512-514. http://dx.doi.org/10.1038/317512a0

[17] Bourdillon, A.J. (1987) Philosophical Magazine Letters, 55, 21-26. http://dx.doi.org/10.1080/09500838708210435 


\section{Appendix. How Many Atoms Are There in a Supercluster Order $p$ ?}

The hierarchic model is an ideal that makes way for defects. Beyond accounting for optimum defocus phase contrast micrographs, such as Figure 1, the second greatest benefit is the facility that the model provides for making meaningful and striking calculations for atomic structure and diffraction. The principal result is the discovery of the generic compromise spacing effect, as a product term in the quasi structure factor calculations. On scanning this term, the peak in the profile yields the measured metric that enables atomic measurements through the new quasi-Bragg law. Atomic sites in the LPS are described in ref. [7].

The simplest way of estimating the number of atoms in a supercluster is to count the number of atomic sites: the unit cell has 13 atoms, each with a $M n$ atom at the center. A cluster is made from 12 cells with $13 \times 12=156$ sites, but with fewer atoms as we shall see. Then, by hierarchic extension, the number of sites in a supercluster order $p$ is $156 \times 12^{\mathrm{p}}$. The number of atoms is less than this.

Start with the cluster: some sites are shared between neighboring cells; some sites a mobile, with neighbors so close that one is vacant; in particular, the central "hole", averted to by Pauling [16], includes several vacancies. Take these three groups one at a time.

Shared sites lie on adjoining edges of neighboring cells. Each cell shares five edges out of 30. Each edge has two $A l$ atoms shared three ways. In all, 60 sites are occupied by only 20 atoms.

Mobile sites contain two sites separated by a distance less than the diameter of metallic $\mathrm{Al}$. Each edge, of the cluster contains 4 sites including one mobile site. There are 120 edge sites, but only 90 atomic sites.

Hole sites occur at the center of the cluster. There are 12 of them on an icosahedron with edge length $1 / \tau$, i.e. smaller than the unit $\mathrm{Al}$ diameter. The sites are like mobile sites, except that they have space for only 3 atoms. Toting the deficits, the cluster has 156-40-30-9=77 atoms.

Proceed to the supercluster, first order. This has 12 clusters in the hierarchic order. The number of sites is $12 \times 77=924$. Dimensions are generally scaled up by $\tau^{2} \approx 2.6$, so that mobile sites do not occur beyond the cluster.

As before, each cluster shares 5 edges. Each edge has $3 \mathrm{Al}$ atoms. So 90 sites are occupied by 45 atoms.

The supercluster has a larger hole than the cluster and this is supposedly filled by interstitials. The space is an icosahedron with edge width $\tau^{3}$. We have previously suggested how the hole could be filled [4] but there is so far no dark field microscopic evidence for support. We estimate that, by contrast with vacancies in the cluster; there is at least a cell of interstitial atoms, i.e. $>13$ atoms. Toting the discrepancies, the supercluster is estimated to contain $924-45+13 \approx 892$ atoms.

By similar considerations, we can proceed to superclusters orders 2, 3, 4, 5, 6 etc. Estimates become increasingly significant with increasing order, and enter into approximations made in the calculations of quasi-structure factors [4] [6]. These take into account shared sites and mobile sites by corresponding computational adjustments on atomic scattering factors; but omit the central holes for fear of introducing errors due to structural misplacements. The numbers derived above lead to the conclusion that there is an increase in atomic populations of about an order of magnitude for each order of supercluster, starting with a population of $\approx 900$ atoms in a first order supercluster. If, more precisely, the ratio $892 / 77=11.6$ is maintained, then a nearer estimate for the atomic populations in a supercluster order $p$ is approximately $892 \times(11.6)^{\mathrm{p}-1}$. Remembering that the atomic scattering factor for $A l$ is about 1.1 for $100 \mathrm{kV}$ electrons, and for $M n$ about 2.1 [12], the atomic populations given are consistent with the values for the squared quasi-structure factors calculated in Figure 3 for the supercluster order 6.

Notice also that the different atomic scattering factors in the two atoms give to the $M n$ atom an intensity increase that is four times greater than $\mathrm{Al}$. This is evident in optimum defocus, phase contrast micrograph in Figure 1.

This treatment raises the issue of stoichiometry. The supercluster has a nominal ratio $A l: M n=6: 1$. The deficits discussed were all on the part of the solute atoms on unit cell edges. However the metastable icosahedral phase grows inside a matrix $A l$ alloy with reduced $M n$ concentration [17]. The depletion in $A l$ in the hierarchic, icosahedral phase, compensates the residual concentration of $M n$ in the matrix. 


\section{Submit or recommend next manuscript to SCIRP and we will provide best service for you:}

Accepting pre-submission inquiries through Email, Facebook, LinkedIn, Twitter, etc.

A wide selection of journals (inclusive of 9 subjects, more than 200 journals)

Providing 24-hour high-quality service

User-friendly online submission system

Fair and swift peer-review system

Efficient typesetting and proofreading procedure

Display of the result of downloads and visits, as well as the number of cited articles

Maximum dissemination of your research work

Submit your manuscript at: http://papersubmission.scirp.org/ 